\title{
Was grenzt das Kompetenzkonzept von etablierten Kategorien wie Fähigkeit, Fertigkeit oder Intelligenz ab?
}

\author{
Oliver Wilhelm • Reinhold Nickolaus
}

Zusammenfassung: Nach einer Konkretisierung des Konzeptes „Kompetenz“ im Rahmen des Schwerpunktprogramms bemühen wir uns um eine Einordnung neuerer kompetenzdiagnostischer Anstrengungen im Lichte etablierter Unterscheidungen. Neben Cronbachs Differenzierung von typischem Verhalten und maximaler Anstrengung und Cattells Unterscheidung von Fragebogen und Testdaten sind Abgrenzungen gegenüber etablierten Oberbegriffen wie etwa „Fähigkeit", „Fertigkeit“, „Talent“ oder auch „Intelligenz“ wünschenswert und erforderlich. Der Nutzen des Kompetenzbegriffs relativ zu etablierten Begriffen sollte zukünftig eindeutig aufgezeigt werden.

Schlüsselwörter: Kompetenz $\cdot$ Fähigkeit $\cdot$ Fertigkeit · Intelligenz

\section{What distinguishes the concept of competence from established categories such as ability, skill or intelligence?}

\begin{abstract}
Following a specification of the concept "competence" as it is applied in the context of the priority program we place recent efforts on competence assessment within established distinctions. Besides Cronbach's differentiation of typical behavior and maximal effort and Cattell's demarcation of test and questionnaire data additional discriminations against broader terms such as "ability", "skill", "talent" but also "intelligence" are desirable and necessary. The utility of the concept of competence relative to established terms needs to be demonstrated unequivocally in the future.
\end{abstract}

Keywords: Ability Competence $\cdot$ Intelligence $\cdot$ Skill

(C) Springer Fachmedien Wiesbaden 2013

Prof. Dr. O. Wilhelm $(\square)$

Institut für Psychologie und Pädagogik, Universität Ulm, 89069 Ulm, Deutschland

E-Mail: oliver.wilhelm@uni-ulm.de

Prof. Dr. R. Nickolaus

Abteilung Berufs-, Wirtschafts- und Technikpädagogik, Universität Stuttgart, Geschwister-Scholl-Straße 24D, 70174 Stuttgart, Deutschland

E-Mail: nickolaus@bwt.uni-stuttgart.de 
Die im Rahmen des Schwerpunktprogramms (SPP) „Kompetenzmodelle zur Erfassung individueller Lernergebnisse und zur Bilanzierung von Bildungsprozessen" vorgenommene bewusste Konzentration auf kognitive Aspekte von Kompetenzen und die damit einhergehende Ausklammerung von Handlungskompetenzen erfolgte - wie bei Fleischer et al. (2013 in diesem Heft) dargestellt - primär unter Zweckmäßigkeitserwägungen und war nicht mit dem Anspruch verbunden, den Kompetenzbegriff generell auf kognitive Aspekte zu begrenzen. Die vorgenommene Fokussierung ist vielmehr mit der Auffassung Weinerts (2001) vereinbar, kognitive und motivationale/volitionale Aspekte von Kompetenzen getrennt zu erforschen. Damit ist auch die Voraussetzung geschaffen, etwaige Zusammenhänge zwischen kognitiven und nicht-kognitiven Kompetenzaspekten zu problematisieren und die Rolle externer Einflüsse (Anforderungen, Aufgaben, Tätigkeiten) zu analysieren. Die Trennung kognitiver und nicht-kognitiver Anteile umschriebener Kompetenzen ist möglicherweise bei sozialen Kompetenzen (z. B. Kommunikationsfähigkeit) weniger funktional als im Bereich fachlicher Kompetenzen. Variationen dieser Trennung bedürfen offensichtlich weiterer empirischer Erforschung. Trotz der Fokussierung auf kognitive Aspekte ist im Rahmen der Untersuchung von Kompetenzen die Abgrenzung motivationaler/volitionaler Aspekte von Kompetenzen (z. B. im Sinne etwaiger metamotivationaler Anstrengungen, die eigene Motivationsentwicklung zu steuern) gegenüber etablierten Selbstberichtsdimensionen jedoch ebenso zu leisten, wie eine weitergehende Klärung geeigneter Operationalisierungen - etwa im Sinne von Persönlichkeitsfähigkeiten (vgl. Riemann 1997).

Kompetenzdiagnostische Verfahren beziehen sich - die hier nicht besprochenen motivationalen Aspekte ausdrücklich ausgeschlossen - im Sinne der auf Cronbach (vgl. 1949) zurückgehenden Unterscheidung von typischem Verhalten und maximaler Anstrengung auf maximale Anstrengung. Es handelt sich bei kompetenzdiagnostischen Verfahren folglich um Instrumente zur Erfassung von Denkleistungen. In aller Regel werden diese Leistungen als latente Fähigkeiten verstanden. Typisches Verhalten wird in der Regel durch Selbstberichte, häufig über Präferenzen und Valenzen erfasst (questionnaire data sensu Cattell 1946). Verfahren, die maximale Anstrengung abbilden, lassen sich wie folgt charakterisieren: a) die untersuchte Person ist sich der Tatsache bewusst, dass ihre Leistung beurteilt wird; b) sie ist willens und in der Lage, maximale Leistung zu zeigen; c) die Bewertungsstandards des Verhaltens sind zur Fällung eines diagnostischen Urteils angemessen (vgl. Sackett et al. 1988). Gerade der zuletzt vorgebrachte Teil der Charakterisierung wird forschungspraktisch durchgängig so umgesetzt, dass zur Verhaltensbeurteilung objektive Leistungsstandards herangezogen werden. Verfahren, die maximale Anstrengung abbilden (test data sensu Cattell 1946), sind - in klarer Abgrenzung zu Instrumenten, die typisches Verhalten abbilden - leistungsbasiert; sie erfordern eine externe Verhaltensbewertung, sie führen in der Regel zu minimalen Antwortverzerrungen, ihre Administration ist langwierig und aufwändig und sie erfassen vorgeblich „Fähigkeiten“, „Fertigkeiten“, „Talente“, „,Begabungen“ oder beispielsweise „Kompetenzen“. Um in der Kompetenzdiagnostik mehr Kohärenz zu stiften, wäre es wünschenswert, wenn die im Sinne kontextspezifischer kognitiver Leistungsdispositionen definierten Kompetenzen (vgl. Fleischer et al. 2013 in diesem Heft) und die zugehörigen Messinstrumente trennscharf von anderen in der Leistungsdiagnostik gebräuchlichen Konzepten unterschieden werden könnten. Zu diesen Konzepten zählen unter anderem die Begriffe Leistung, 
Fähigkeit, Begabung, Eignung, Fertigkeit, Tüchtigkeit, Talent und Performanz sowie die Begriffe Lernen, Wissen und Intelligenz. Zum Teil sind diese Begriffe weitgehend redundant (etwa Fähigkeit und Begabung) und - noch entscheidender - die Begriffe eignen sich nicht für eine trennscharfe Klassifikation vorhandener Tests (vgl. Bond 1989) oder von Messansätzen - ein Umstand der voraussichtlich auch auf Kompetenztests zutrifft. Tatsächlich reflektieren diese Konzepte in erster Linie separierte Forschungstraditionen zu kognitiven Leistungen, deren verstärkte Zusammenführung in zukünftigen Forschungsanstrengungen wünschenswert wäre (vgl. Snow und Lohman 1984). Das setzt allerdings begriffliche Schärfungen und empirische Untersuchungen zu bestehenden Zusammenhängen voraus.

Der Begriff „Lernen“ kann als ein Prozess kognitiver Veränderungen verstanden werden und referiert daher - in Abgrenzung zum Begriff „Kompetenz“ - nicht auf Dispositionskonstrukte oder individuelle Unterschiede in latenten Verhaltensbereitschaften (siehe jedoch die Arbeiten zur Lernfähigkeit, etwa von Guthke und Beckmann 2001). Auf eine Abgrenzung zwischen „Kompetenz“ und „Lernen“ (im Sinne des Prozess des Lernens) wird daher verzichtet.

Für eine Abgrenzung gegenüber „Wissen“ als einem möglichen Produkt des Lernens ergibt sich die Schwierigkeit, dass der Wissensbegriff variantenreich Verwendung findet. Zur Vermeidung von Missverständnissen bei der Verwendung des Terminus Wissen könnte der Begriff der Kompetenz als die Einheit von Wissen und Können gefasst werden. Hinsichtlich dieser Abgrenzung wäre kritisch zu erörtern, inwiefern derzeit gebräuchliche Kompetenzoperationalisierungen tatsächlich vorrangig deklaratives und prozedurales Wissen aus einem jeweils umschriebenen Gegenstandsbereich erfassen.

Für eine Abgrenzung zum Intelligenzbegriff ist entscheidend, dass Intelligenz als Oberbegriff im Gegensatz zu Kompetenz weniger auf Domänen fokussiert ist. Allerdings werden in gängigen Intelligenzstrukturmodellen durchaus domänenspezifische Konstrukte wie etwa „Visualisierung“ postuliert. Ob und inwiefern Intelligenzkonstrukte relativ zu Kompetenzkonstrukten als zeitstabilere und weniger erwerbbare Dispositionen gelten können, ist eine empirische Frage. Die Erforschung von Mechanismen der Veränderbarkeit der untersuchten Denkleistungen könnte ein wichtiger Schlüssel für ein vertieftes Verständnis des Untersuchungsgegenstandes sein. Bezieht man in die Abgrenzungsüberlegungen kristalline Intelligenz ein, so sind weitgehende Überschneidungen zwischen Intelligenz und Kompetenz die Folge. Bei einer Beschränkung auf fluide Intelligenz ist eine Abgrenzung gegenüber dem Kompetenzbegriff dagegen relativ unproblematisch: Bei Intelligenzmessungen handelt es sich im Wesentlichen um weitgehend dekontextualisierte Denkleistungen (vgl. Ackerman und Beier 2003; Wilhelm 2005). Zu berücksichtigen ist, dass gängige Intelligenztests vermutlich kaum geeignet sind, das in spezifischen Domänen erworbene Wissen und damit assoziierte Befähigungen im Handeln adäquat zu erfassen. Genau darauf sind hingegen die Bemühungen bei der Entwicklung von Kompetenztests ausgerichtet. Die Verortung mutmaßlich spezifischer Kompetenzen in einem Netzwerk weiterer Denkleistungen - darunter auch ähnlich konzeptualisierte Kompetenzen - ist ein unverzichtbarer und inhaltlich kritischer Bestandteil zukünftiger Forschung bei der Etablierung möglicherweise neuer Kompetenzen (vgl. Wilhelm 2009).

Die derzeit noch zu beklagenden Schwierigkeiten bei der Etablierung spezifischer Kompetenzen als Denkleistungskonstrukte haben mehrere Quellen. Einerseits ist für die 
Etablierung eines Denkleistungskonstruktes mehr erforderlich als hinreichend inspirierte Bemühungen im Rahmen der Testkonstruktion. Überzeugende empirische Belege, etwa über die differenzielle Veränderbarkeit durch maßgeschneiderte Instruktionen oder die inkrementelle Validität bei der Vorhersage relevanter Bildungsereignisse, müssen hinzukommen, um einer postulierten Kompetenz wissenschaftliche Glaubwürdigkeit zu verleihen. Andererseits darf bei den Abgrenzungsbemühungen gegenüber Feldern wie der Intelligenzdiagnostik nicht übersehen werden, dass dort bei der Auswahl von Testaufgaben gerade nicht inhaltliche, sondern ganz vorrangig empirische Belege verwendet werden. Daher ist es nicht überraschend, dass etwa Untertests zu quantitativen Fähigkeiten oft Itemmaterial enthält, das ohne weitere Überarbeitungen auch in mathematischen Kompetenztests eingesetzt werden könnte.

Danksagung: Diese Veröffentlichung wurde ermöglicht durch Sachbeihilfen der Deutschen Forschungsgemeinschaft (Kennz.: WI 2667/7-1 und Ni 606/3-2 (kooptiertes Projekt)) im Schwerpunktprogramm „Kompetenzmodelle zur Erfassung individueller Lernergebnisse und zur Bilanzierung von Bildungsprozessen“" (SPP 1293).

\section{Literatur}

Ackerman, P. L., \& Beier, M. E. (2003). Trait complexes, cognitive investment, and domain knowledge. In R. J. Sternberg \& E. L. Grigorenko (Hrsg.), Perspectives on the psychology of abilities, competencies, and expertise (S. 1-30). New York: Cambridge University Press.

Bond, L. (1989). The effects of special preparation on measures of scholastic ability. In R. L. Linn (Hrsg.), Educational measurement (3. Aufl., S.429-444). New York: American Council on Education/Macmillan.

Cattell, R. B. (1946). Description and measurement of personality. New York: World Book.

Cronbach, L. J. (1949). Essentials of psychological testing. New York: Harper.

Fleischer, J., Koeppen, K., Kenk, M., Klieme, D., \& Leutner, D. (2013). Kompetenzmodellierung: Struktur, Konzepte und Forschungszugänge des DFG-Schwerpunktprogramms. In D. Leutner, E. Klieme, J. Fleischer \& H. Kuper (Hrsg.), Kompetenzmodelle zur Erfassung individueller Lernergebnisse und zur Bilanzierung von Bildungsprozessen: aktuelle Diskurse im DFGSchwerpunktprogramm (18. Sonderheft der Zeitschrift für Erziehungswissenschaft, DOI: 10.1007/s11618-013-0379-z). Wiesbaden: VS Verlag für Sozialwissenschaften.

Guthke, J., \& Beckmann, J. F. (2001). Intelligenz als „Lernfähigkeit“ - Lerntests als Alternative zum herkömmlichen Intelligenztest. In E. Stern \& J. Guthke (Hrsg.), Perspektiven der Intelligenzforschung. Ein Lehrbuch für Fortgeschrittene (S. 137-161). Lengerich: Pabst.

Riemann, R. (1997). Persönlichkeit: Fähigkeiten oder Eigenschaften? Lengerich: Pabst Science.

Sackett, P. R., Zedeck, S., \& Fogli, L. (1988). Relations between measures of typical and maximum job performance. Journal of Applied Psychology, 73, 482-486.

Snow, R. E., \& Lohman, D. F. (1984). Toward a theory of cognitive aptitude for learning from instruction. Journal of Educational Psychology, 76, 347-376.

Weinert, F. E. (2001). Concept of competence: A conceptual clarification. In D. S. Rychen \& L. H. Salganik (Hrsg.), Defining and selecting key competencies (S. 45-65). Seattle: Hogrefe.

Wilhelm, O. (2005). Measuring reasoning ability. In O. Wilhelm \& R. W. Engle (Hrsg.), Understanding and measuring intelligence (S.373-392). London: Sage.

Wilhelm, O. (2009). Issues in computerized ability measurement: Getting out of the jingle and jangle jungle. In F. Scheuermann \& A. G. Pereira (Hrsg.), The transition to computerbased assessment (S. 145-150). Luxembourg: Office for Official Publications of the European Communities. 